\title{
Iron Deficiency Anemia in Oral Submucous Fibrosis
}

\author{
${ }^{1}$ Richa Saxena, ${ }^{2}$ Monisha Batra
}

\begin{abstract}
Oral submucous fibrosis (OSMF) is an insidious, chronic disease affecting any part of the oral cavity and rarely pharynx. It has a high malignant transformation rate. Juxta epithelial inflammatory reaction with subsequent fibroelastic changes of lamina propria can be seen frequently with OSMF. It may cause significant abnormalities of blood such as anemia and a decline in serum iron levels. Therefore, various studies have been conducted which correlate the hemoglobin and iron serum levels in a patient suffering from OSMF. This article discusses the association between iron deficiency anemia and OSMF and how it can act as a marker for early diagnosis and prognosis of this condition.
\end{abstract}

Keywords: Grading, Hemoglobin staging, Iron deficiency anemia, Malignant transformation, Oral submucous fibrosis, Serum iron.

How to cite this article: Saxena R, Batra M. Iron Deficiency Anemia in Oral Submucous Fibrosis. World J Anemia 2018;2(2):51-54.

\section{Source of support: Nil}

Conflict of interest: None

\section{INTRODUCTION}

Oral submucous fibrosis (OSMF) is an uncommon disease which is considered to be a precancerous condition. OSMF is a chronic, progressive, and irreversible disease of unknown etiology. It involves oral, oropharyngeal, and sometimes esophageal mucosa. The earliest description of the disease was by Schwartz in 1952, ${ }^{1}$ who coined the term atrophica idiopathic mucosa oris. It has also been termed as idiopathic scleroderma of mouth, idiopathic palatal fibrosis, and sclerosing stomatitis. ${ }^{2}$

The OSMF predominant in India, Bangladesh, Sri Lanka, Pakistan, Taiwan, Southern China, Polynesia, and Micronesia. Several case series are also reported among Asians settling in the UK and South and East Africa. ${ }^{3}$

The prevalence of OSMF is chiefly seen in the younger generation, and it is increasing further especially in India

\footnotetext{
${ }^{1}$ Obstetrician Gynecologist, ${ }^{2}$ Postgraduate Student,

1Jaypee Brothers Medical Publishers, New Delhi, India

${ }^{2}$ Santosh Dental College, Ghaziabad, Uttar Pradesh, India
}

Corresponding Author: Richa Saxena, Obstetrician Gynecologist, Jaypee Brothers Medical Publishers, New Delhi, India, e-mail: synapse94@hotmail.com and Southeast Asian countries. The various etiological factors that initiate the disease process include chewing of areca nut, any nutritional deficiency, immunologic processes, and genetic predisposition. ${ }^{4,5}$

Deficiencies caused mainly due to iron and vitamins are considered as one of the etiological factors of OSMF. Iron plays an important role in maintaining the complete integrity and health of epithelia of the digestive tract and its contribution to normal enzymatic functions.

Oral submucous fibrosis is considered as an Asian version of sideropenic dysphagia, where prolonged iron deficiency can lead to mucosal susceptibility to chilies and areca nut products which act as irritants. ${ }^{6}$

Hemoglobin levels, particularly serum iron levels, are considered as biochemical indicators for nutritional assessment. ${ }^{7}$ Deficiencies of vitamin $B^{12}$, folate, and iron affect the effectiveness of oral mucosa.

Various hematological abnormalities that have been reported in OMSF include:

- Increase in blood sedimentation rate

- Anemia

- Decrease in serum iron levels

- Increase in total iron binding capacity (TIBC). ${ }^{8}$

\section{Etiological Factors ${ }^{2}$}

- Chilies: Use of chilies plays an important etiological role in OSMF. They contain 8-methyl-6-nonenoic acid that is the main irritant.

- Tobacco: It is the most common factor involved in oral malignancy and acts as a local irritant in OSMF.

- Lime: It is consumed along with betel nut chewing. This causes vesicle and ulcer formation in the oral mucosa of susceptible people.

- Betel nut: It is most commonly used as a psychotropic and antihelminthic agent. It is also used as an aftermeal digestant to ease abdominal discomfort. ${ }^{1}$

- Nutritional deficiency: A subclinical vitamin B complex deficiency has been noted in cases suffering from OSMF.

- Bacterial infections: Streptococcal toxicity can cause OSMF.

- Defective iron metabolism: Microcytic hypochromic anemia with high serum iron has been reported in individuals suffering from OSMF.

- Collagen disorders: It is localized collagen disease of the oral cavity which has been linked to scleroderma, rheu- 
matoid arthritis, intestinal fibrosis, and Dupuytren's contracture.

- Immunological disorders: Raised erythrocyte sedimentation rate is indicative of an immunological disorder. Further, there is a rise in levels of immunoglobulin $\mathrm{A}$ (IgA), IgG, and IgM. These increased levels indicate an antigenic stimulus in the absence of any infection.

- Altered salivary composition: Saliva analysis of patients suffering from OSMF shows an increase in $\mathrm{pH}$, salivary amylase, alkaline phosphatase, and potassium levels, and low levels of calcium.

\section{Clinical Features $^{2}$}

- The OSMF mainly affects the population between the age group of 20 years and 40 years.

- It affects both the sexes.

- Most frequent locations are buccal mucosa and retromolar areas. Also involves soft palate, uvula, tongue, labial mucosa, and palatal fauces.

- Symptoms

- Burning sensation of the oral mucosa

- Hypersalivation or dryness of mouth

- Vesiculation

- Ulceration

- Pigmentation

- Recurrent stomatitis

- Defective gustatory sensation.

- Signs

- Gradual stiffening of the oral mucosa

- Difficulty in the opening of the mouth

- Blanching of mucosa

- Leathery mucosa

- Difficulty in swallowing of food.

\section{Grading $^{9}$}

Pindborg and Sirsat were the first to classify OSMF according to their histopathological features:

- Very early stage: Fibrillar collagens are dispersed, and there is marked edema. Plump young fibroblasts can be seen with abundant cytoplasm. Dilated blood vessel along with inflammatory cells especially polymorphonuclear leukocytes and eosinophils can be seen.

- Early stage: Early hyalinization can be seen along with plump and young fibroblasts and inflammatory cells such as eosinophills, lymphocytes, and occasionally plasma cells.

- Moderately advanced stage: Collagen is moderately hyalinized. Thick collagen bundles can be seen along with normal blood vessels and the presence of lymphocytes and plasma cells can be seen.

- Advanced stage: Complete hyalinization of collagen is seen. There is an absence of edema. Fibroblasts are not seen in the hyalinized areas along with obliterated blood vessels.

\section{Staging ${ }^{10}$}

Staging of OSMFis mainly of two types (Table 1):

- Clinical staging: Based on the location of the bands in the mouth.

- Functional staging: Amount of mouth opening.

\section{Malignant Transformation ${ }^{2}$}

Oral submucous fibrosis can transform into malignancy without predilection to any specific site or intraoral region. The various changes that can be noted while this transformation includes:

Atrophic epithelium becomes hyperkeratotic

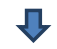

Basal cell hyperplasia and intracellular edema develops

Epithelial atypia can be seen along with moderate epithelial hyperplasia

Carcinoma can develop

Further, according to the World Health Organization (WHO) collaborating center for oral precancerous lesions, it is not always conclusive that OSMF may predispose to oral cancer. But there is a high probability of such a relationship, which is based on the following facts:

- The incidence rate of oral leukoplakia is 6-8 times greater than in patients having OSMF as compared to the control group.

- Alterations seen in the immunity of cancer patients are similar to that of patients suffering from OSMF.

Table 1: Functional and clinical staging of oral submucous fibrosis

Clinical staging
Stage 1: Bands seen only on the soft palate, the base of the
tongue, and the palatine arches
Stage 2: Bands seen on the soft palate, base of the tongue,
palatine arches, and the buccal mucosa

Stage 3: Bands seen on the soft palate, base of the tongue, palatine arches, buccal mucosa, and labial mucosa
Functional staging

Stage A: When the mouth opening is around $20 \mathrm{~mm}$

Stage $B$ : When the mouth opening is around $11-19 \mathrm{~mm}$

Stage C: When the mouth opening is around $10 \mathrm{~mm}$ 
Cancer patients having OSMF have exceeded $1.2 \%$ of people having submucous fibrosis in general population.

\section{Relationship between Iron Deficiency Anemia and Oral Submucous Fibrosis}

A number of studies have been conducted worldwide to build a relationship between iron deficiency anemia and OSMF.

Ganpathy et al. ${ }^{11}$ conducted a study which constituted 20 patients who were clinically diagnosed with OSMF and a control group, which included 10 healthy subjects and another 10 patients with iron deficiency anemia and without any history of areca-nut chewing constituted the anemic group. Serum and hemoglobin levels were tested with Ferrene and Sahli's method, respectively. This study reported lower levels of hemoglobin and serum iron in those diagnosed with oral submucous fibrosis as compared to those of the control group. Similar results were reported by studies conducted by Dhanasekaran and Devi, ${ }^{12}$ Maher et al., ${ }^{13}$ Khanna and Karjodkar, ${ }^{14}$ and Taneja et al.. ${ }^{15}$ Lower levels of hemoglobin and serum iron are indicative of iron deficiency anemia. ${ }^{16}$ Iron deficiency anemia in patients with OSMFmay be due to the precancerous nature of this condition.

Another study conducted by Hegde et al. ${ }^{17}$ comprised 60 patients who were clinically and histopathologically diagnosed with OSMF. Clinically, the OSMF group was staged into four main stages, i.e., stages I-IV. Fifteen healthy individuals were included which acted as a control group and another group included 15 patients with iron deficiency anemia. Hemoglobin and serum iron level was measured as done in the above study. The study showed a considerable drop in the levels of mean hemoglobin and mean serum iron in OSMF group as compared to the control group. Further on comparing between the OSMF group and the anemic group, it was reported hemoglobin and mean serum iron levels were significantly lower in the anemia group as compared to the OSMF group. Also, as the clinical stage of OSMF advanced from stage I to stage IV, there was a major fall in the hemoglobin and serum iron levels.

\section{DISCUSSION}

Oral submucous fibrosis is a chronic, insidious oral mucosal condition which occurs predominantly in younger individuals of Asia and India. In India alone, around 5 million people are suffering from OSMF. ${ }^{18}$

The incidence rate of OSMF is increasing in India due to the high demand of areca nut in the form of pan masala and increase in the uptake of this habit by young people due to easy access and good market strategies. ${ }^{19}$
Although iron deficiency anemia and OSMF are two different conditions, the clinical picture of OSMF depicts that of iron deficiency anemia with signs and symptoms such as blanching, burning sensation, and difficulty in swallowing.

\section{CONCLUSION}

This article provides the pieces of evidence from the studies conducted by various authors establishing the relationship between iron deficiency anemia and OSMF. According to the studies mentioned above, the biochemical assessment of oral precancerous conditions such as OSMF may help in its early diagnosis and prognosis. Further, iron therapy should be administered consecutively along with a properly balanced diet along with other treatment modalities.

\section{REFERENCES}

1. Sabharwal R, Gupta S, Kapoor K, Puri A, Rajpal K, Sabharwal R. Oral submucous fibrosis: A review. J Adv Med Dent Scie Res 2013;1(1):29-37.

2. GhomAG, Shubhangi M. Premalignant Lesions and Conditions. Textbook of Oral and Maxillofacial Pathology, 2nd edition. New Delhi: Jaypee Brothers Medical Publishers (P) Ltd; 2013. pp. 243-248.

3. IARC Working Group on the Evaluation of Carcinogenic Risks to Humans. Betel-quid and areca-nut chewing and some areca-nut derived nitrosamines. IARC Monogr Eval Carcinog Risks Hum 2004;85:123-129.

4. Cox SC, Walker DM. Oral submucous fibrosis: A review. Aust Dent J 1996;41(5):294-299.

5. Canniff JP. Oral submucous fibrosis: Its pathogenesis and management. Br Dent J 1986;160:429.

6. Rajendran R, Vijayakumar T. An alternative pathogenetic pathway for oral submucous fibrosis. Medical Hypotheses 1989;30:35-37.

7. Khanna S. Immunological and biochemical markers in oral carcinogenesis: The public health perspective. Int J Environ Res Public Health 2008;5:418-422.

8. Rajendran R. Oral submucous fibrosis. J Oral Maxillofac Pathol 2003;7:1-4.

9. More CB, Gupta S, Joshi J, Varma SN. Classification system for oral submucous fibrosis. Journal of Indian Academy of Oral Medicine and Radiology 2012;24(1):24-29.

10. Haider SM, Merchant AT, Fikree FF, Rahbar MH. Clinical and functional staging of oral submucous fibrosis. British Journal of Oral and Maxillofacial Surgery 2000 Feb 1;38(1):12-15.

11. Ganapathy KS, Gurudath S, Balikai B, Ballal S, Sujatha D. Role of iron deficiency in oral submucous fibrosis: An initiating or accelerating factor. Journal of Indian Academy of Oral Medicine and Radiology 2011;23(1):25-28.

12. Anuradha CD, Shyamala Devi CS. Serum protein, ascorbic acid and iron tissue collagen in oral submucous fibrosis: A preliminary study. Indian J Med Res 1993;98:147-151.

13. Maher R, Aga P, Johnson NW, Sankaranarayanan R, Warnakulasuriya S. Evaluation of multiple micronutrient supplementation in the management of oral submucous fibrosis in Karachi, Pakistan. Nutrition and Cancer 1997;27:41-47. 
14. Khanna SS, Karjodkar FR. Circulating immune complexes and trace elements (copper, iron and selenium) as markers in oral precancer and cancer: A randomized, controlled clinical trial. Head Face Med 2006;2:33.

15. Taneja L, Bagewadi A, Keluskar V. Hemoglobin levels in patients with oral submucous fibrosis. JIAOMR.2007;19:329-333.

16. DeRossi Scott S, Sree R. Anemia. OOOE 2003;95:131-141.

17. Hegde K, Gharote H, Nair P, Agarwal K, Saawarn N, Rajaram DK. Iron deficiency in oral submucous fibrosis: accelerator or a promoter? International Journal of Oral and Maxillofacial Pathology 2012 Feb 26;3(1):2-7.

18. Angadi PV, Rao SS. Areca nut in pathogenesis of oral submucous fibrosis: revisited. Oral Maxillofac Surg. 2011; 15:1-9.

19. Ranganathan K, Devi MU, Joshua E, Kirankumar K, Saraswathi TR. Oral submucous fibrosis: a case control study in Chennai, South India. Journal of Oral Pathology \& Medicine 2004 May;33(5):274-277. 